\title{
BIORTHOGONAL MULTIRESOLUTION ANALYSES AND DECOMPOSITIONS OF SOBOLEV SPACES
}

\author{
ABDELLATIF JOUINI and KHALIFA TRIMÈCHE
}

(Received 25 September 2000 and in revised form 1 February 2001)

\begin{abstract}
The object of this paper is to construct extension operators in the Sobolev spaces $\left.\left.H^{k}(]-\infty, 0\right]\right)$ and $H^{k}([0,+\infty[)(k \geq 0)$. Then we use these extensions to get biorthogonal wavelet bases in $H^{k}(\mathbb{R})$. We also give a construction in $L^{2}([-1,1])$ to see how to obtain boundaries functions.
\end{abstract}

2000 Mathematics Subject Classification. 41A58, 42C15.

1. Introduction. The decomposition method was used by Ciesielski and Figiel $[1,2]$ to construct spline bases of general Sobolev spaces $W_{p}^{k}(M)(k \in \mathbb{Z}$ and $1 \leq p \leq+\infty)$ where $M$ is a compact Riemannian manifold of dimension $d$. The extension operators constructed by Ciesielski and Figiel are based on the extension theorem of Stein [13] which do not permit to get multiresolution with compact support.

In 1993, we have constructed biorthogonal wavelet bases in $\Omega$ which is an interval and a bounded open set of $\mathbb{R}^{2}$ (see $[8,9]$ ). These bases are adapted to study Sobolev spaces $H^{1}(\Omega)$ and $H_{0}^{1}(\Omega)$.

Recently, in 1997, the decomposition was used by Cohen, Dahmen, and Schneider (see $[3,4,5]$ ) to construct biorthogonal wavelet bases $\left(\psi_{\lambda}, \tilde{\psi}_{\lambda}\right)_{\lambda \in \nabla}$ of $L^{2}(\Omega)$ where $\Omega$ is a bounded domain of $\mathbb{R}^{d}$. These bases were those of Sobolev spaces $H^{s}(\Omega)$ for $s$ only in the interval ] $-3 / 2,3 / 2[$.

There are related constructions given by Masson in [11]. All these constructions are based on the decomposition method; there is a slight difficulty in their presentation, due to notational burden. Moreover, it is unclear how to obtain regularity Sobolev estimates for $|s| \geq 3 / 2$ and also to get associated fast algorithms.

In this paper, we use a direct method based on the result described in [9] to define orthogonal and biorthogonal multiresolution analyses on the interval $[0,1]$ which are generated by a finite number of basis functions. These analyses are regular and have a compact support. Next we use a decomposition method to construct segmented biorthogonal multiresolution analyses in $\mathbb{R}$. In this case, we get decompositions of the Sobolev space $H^{k}(\mathbb{R})(k \in \mathbb{Z})$ by using simple extension operators. These extensions permit to get fast algorithms for associated biorthogonal multiresolution analyses because all bases constructed in this paper satisfy the lifting scheme described in $[11,14]$ in order to get wavelet bases with compact support and with the same regularity as for Daubechies bases [6]. This analysis is adapted to the study of regular functions in $H^{k}\left(\left[0,+\infty[), H^{k}(]-\infty, 0\right]\right)$, and $H^{k}(\mathbb{R})$, by using extensions. We also give a construction of segmented biorthogonal multiresolution analysis in $L^{2}([-1,1])$ to 
see how to obtain boundary functions. Finally, recall that segmented multiresolution analyses are useful in many applications as numerical simulation for elliptic problems or image processing (see [11]).

The first object of this paper is studied in Section 2. In fact, we construct biorthogonal multiresolution $\left(V_{j}, V_{j}^{*}\right)(j \in \mathbb{Z})$ on the interval $[0,1]$. By a derivation on $V_{j}$ and an integration on $V_{j}^{*}$, we get biorthogonal multiscale analysis $\left(V_{j}^{(1)}, V_{j}^{(-1)}\right)$ of the space $L^{2}([0,1])$. Let $P_{j}$ be the projector on $V_{j}$ parallel to $\left(V_{j}^{*}\right)^{\perp}$ and $P_{j}^{(1)}$ be the projector in $V_{j}^{(1)}$ parallel to $\left(V_{j}^{(1)}\right)^{\perp}$, then we have the following commutation property:

$$
\frac{d}{d x} \circ P_{j}=P_{j}^{(1)} \circ \frac{d}{d x} .
$$

If the multiscale function $\varphi$ is regular, we develop a similar strategy for constructing biorthogonal multiresolution analysis $\left(V_{j}^{(d)}, V_{j}^{(-d)}\right)(d \in \mathbb{N})$ of the space $L^{2}([0,1])$. Moreover, we have the commutation property between scale projectors and derivation

$$
\frac{d}{d x} \circ P_{j}^{(d-1)}=P_{j}^{(d)} \circ \frac{d}{d x} .
$$

The biorthogonal analysis $\left(V_{j}^{(d)}, V_{j}^{(-d)}\right)$ are adapted to the study of Sobolev spaces $H^{k}([0,1])$ and $H_{0}^{k}([0,1])$ for $k \in \mathbb{Z}$.

Section 3 is devoted to the construction of extension operators. We show that if we consider an extension operator $E$ from $\left.\left.H^{k}(]-\infty, 0\right]\right)$ into $H^{k}(\mathbb{R})$ for $k \in \mathbb{Z}$, we get decomposition of the Sobolev space $H^{k}(\mathbb{R})$ by using an isomorphism between the space $H^{k}(\mathbb{R})$ and the space $\left.\left.E\left(H^{k}(]-\infty, 0\right]\right)\right)+H_{0}^{k}([0,+\infty[)$. This isomorphism permits to get biorthogonal multiresolution analysis of $H^{k}(\mathbb{R})$ based on those of $\left.\left.L^{2}(]-\infty, 0\right]\right)$ and $L^{2}([0,+\infty$ [). These multiscale analyses satisfy the commutation property between scale projectors and derivation. All wavelet bases constructed in this section have a compact support and are adapted to higher regularity analysis.

In conclusion, we describe "new" biorthogonal multiresolution analysis in $L^{2}$ $([-1,1])$ to show more clearly how to construct boundary functions. These analyses are adapted to the study of the Sobolev spaces $H^{1}([-1,1])$ and $H_{0}^{1}([-1,1])$.

2. Multiresolution analyses on the interval and applications. Recall that multiresolution analyses (denoted by MRA) on the interval are introduced by Meyer [12]. For other related constructions see [7, 8, 9]. In the first part of this section, we construct orthogonal multiresolution analyses $V_{j}$ on the interval $[0,1]$ and we show that there exists a new supplement $X_{j}$ of $V_{j}$ in $V_{j+1}$. In the second part, we introduce biorthogonal multiresolution analyses on the interval and we prove, by using a derivation, that we get other biorthogonal multiresolution analyses which are adapted to the study of Sobolev spaces $H^{k}([0,1])$ and $H_{0}^{k}([0,1])$ for $k \in \mathbb{Z}$. Moreover, we get the commutation property between scale projectors and derivation. These analyses have compact support and are adapted to higher regularity analysis. They will be used in the next section and, by using some natural extensions, we get segmented biorthogonal analyses.

2.1. Orthogonal multiresolution analyses on the interval. We recall that the orthogonal multiresolution analysis (denoted by OMRA) $V_{j}(\mathbb{R})$ of Daubechies [6] satisfies 
the following properties:

- $V_{0}$ has an orthonormal base $\varphi(x-k), k \in \mathbb{Z}$, where $\varphi$ the scaling function with compact support.

- $\varphi(x / 2)=\sum_{k=0}^{2 N-1} a_{k} \varphi(x-k)$, the sequence of real numbers $\left(a_{k}\right)$ satisfies $a_{0} \neq 0$ and $a_{2 N-1} \neq 0$. Moreover, we have $\hat{\varphi}(2 \xi)=M_{0}\left(e^{-i \xi}\right) \hat{\varphi}(\xi)$ where $M_{0}\left(e^{-i \xi}\right)=\sum_{k=0}^{2 N-1} a_{k} e^{-k i \xi}$ and " $\wedge$ " is the classical Fourier transform on $\mathbb{R}$.

- $\operatorname{supp} \varphi=[0,2 N-1]$.

- The associated wavelet $\psi$ is defined by

$$
\hat{\psi}(2 \xi)=e^{-i(2 N-1) \xi} M_{0}\left(-e^{-i \xi}\right) \hat{\varphi}(\xi) .
$$

- $W_{0}(\mathbb{R})$ (the orthogonal complement of $V_{0}(\mathbb{R})$ in $V_{1}(\mathbb{R})$ ) has an orthonormal base $\psi(x-k), k \in \mathbb{Z}$.

- $V_{j}(\mathbb{R})$ has an orthonormal base $\varphi_{j, k}(x)=2^{j / 2} \varphi\left(2^{j} x-k\right), j, k \in \mathbb{Z}$, and $W_{j}(\mathbb{R})$ has an orthonormal basis $\psi_{j, k}(x)=2^{j / 2} \psi\left(2^{j} x-k\right), j, k \in \mathbb{Z}$.

The multiresolution of Daubechies is orthogonal in $L^{2}(\mathbb{R})$, but if we take its restriction to $[0,1]$, we do not get an orthogonal multiresolution analysis in $L^{2}([0,1])$. Moreover, if we consider the functions $\varphi_{j, k}(x)_{/[0,1]}$, we have an independent system but not orthogonal. However, if we consider the functions $\psi_{j, k}(x)_{/[0,1]}$ we get a dependent system (see [12]). Then, the construction of orthogonal multiresolution analyses in $[0,1]$ (or biorthogonal) is technical specially near the boundaries 0 and 1 .

In the following, we have to construct new orthogonal wavelet bases in $[0,1]$. For this purpose, we consider the OMRA $V_{j}(\mathbb{R})$ of Daubechies and we denote

$$
\begin{aligned}
& V_{j}([0,1])=\operatorname{Vect}\left\{\varphi_{j, k} /[0,1], \varphi_{j, k} \in V_{j}(\mathbb{R})\right\}, \\
& v_{j}([0,1])=\operatorname{Vect}\left\{\varphi_{j, k}, \operatorname{supp} \varphi_{j, k} \subset[0,1]\right\} .
\end{aligned}
$$

DEFINITION 2.1. A sequence $\left\{V_{j}\right\}_{j \geq j_{0}}$ of closed subspaces of $L^{2}([0,1])$ is called a MRA on $L^{2}([0,1])$ associated with $V_{j}(\mathbb{R})$ if we have

(i) for all $j \geq j_{0}, v_{j}([0,1]) \subset V_{j} \subset V_{j}([0,1])$;

(ii) for all $j \geq j_{0}, V_{j} \subset V_{j+1}$.

EXAMPLE 2.2 (periodic wavelets). For $j \geq 0$, we denote

$$
\tilde{\varphi}(x)=\sum_{p \in \mathbb{Z}} \varphi_{j, k}(x-p)=\sum_{p \in \mathbb{Z}} \varphi_{j, k+p 2^{j}}(x) .
$$

Then $V_{j}$ is defined as the space generated by the functions $\tilde{\varphi}_{j, k /[0,1]}$. We then get periodic wavelet bases which are adapted to the study of periodic regular functions.

The following result of Meyer [12] gives another example of MRA of $L^{2}([0,1])$ which is important to establish the first goal of this paper.

LEMMA 2.3. For $j \geq j_{0}$, the functions $\varphi_{j, k /[0,1]}, 2-2 N \leq k \leq 2^{j}-1$, form a Riesz basis of $V_{j}([0,1])$ and the functions $\psi_{j, k /[0,1]},-N+1 \leq k \leq 2^{j}-N$, constitute a Riesz basis of $W_{j}([0,1])$. 
We describe a complement (not orthogonal) of $V_{j}$ in $V_{j+1}$. More precisely, we have the following important result from [9].

Proposition 2.4. Let $j_{0}$ be the smallest integer satisfying $2^{j_{0}} \geq 4 N-4$. For $j \geq j_{0}$, we denote

$$
\begin{gathered}
X_{j}=\operatorname{Vect}\left\{\psi_{j, k}, 0 \leq k \leq 2^{j}-2 N+1 ; \varphi_{j+1,2 k+1}, 0 \leq k \leq N-2 ;\right. \\
\left.\varphi_{j+1,2 k},-2 N+2 \leq k \leq 2^{j}-N\right\} .
\end{gathered}
$$

Then

(i) $\operatorname{dim} X_{j}=2^{j}$.

(ii) There exists an integer $J$ such that for all $j \geq J, V_{j+1}=V_{j} \oplus X_{j}$.

PROoF. First we remark that $\operatorname{supp} \psi_{j, k} \subset[0,1]$ for $0 \leq k \leq 2^{j}-2 N+1, \operatorname{supp} \varphi_{j+1,2 k+1}$ $\subset\left[0,(4 N-4) / 2^{j+1}\right]$ for $0 \leq k \leq N-2$ and $\operatorname{supp} \varphi_{j+1,2 k} \subset\left[-(4 N-4) / 2^{j+1}, 1-1 / 2^{j+1}\right]$ for $-2 N+2 \leq k \leq 2^{j}-N$. We denote by $x_{j, k},-N+1 \leq k \leq 2^{j}-N$, the generated system of $X_{j}$ defined by

- for $-N+1 \leq k \leq-1, x_{j, k}=\varphi_{j+1,2(k+N-1)+1}$;

- for $0 \leq k \leq 2^{j}-2 N+1, x_{j, k}=\psi_{j, k}$;

- for $2^{j}-2 N+2 \leq k \leq 2^{j}-N, x_{j, k}=\varphi_{j+1,2 k}$.

The matrix $\left(m_{p, q}\right)$, with $-N+1 \leq p, q \leq 2^{j}-1$ of coefficients of $x_{j, k}$ relatively to $\psi_{j, k /[0,1]}$ (we decompose $x_{j, k}$ with respect to $\varphi_{j, k /[0,1]}$ and $\psi_{j, k /[0,1]}$ and we take only the coefficients corresponding to $\left.\psi_{j, k[0,1]}\right)$ is defined by

$$
\left[\begin{array}{ll}
A & 0 \\
0 & B
\end{array}\right],
$$

where $A=\left(m_{p, q}\right)$, with $-N+1 \leq p, q \leq 2^{j-1}-1$, is a superior triangular matrix with all its diagonal coefficients are different from zero and $B=\left(m_{p, q}\right)$, with $2^{j}-N+1 \leq$ $p, q \leq 2^{j}-N$, is an inferior triangular matrix with all its diagonal coefficients are different from zero. The diagonal terms of $A$ and $B$ are defined by

$$
\begin{aligned}
m_{p, p}= & \frac{1}{\sqrt{2}}\left\langle\varphi(x-2 N+1), \psi\left(\frac{x}{2}\right)\right\rangle, \quad \text { if } p \leq-1, \\
& m_{p, p}=1, \quad \text { if } 0 \leq p \leq 2^{j}-2 N+1, \\
m_{p, p}= & \frac{1}{\sqrt{2}}\left\langle\varphi(x), \psi\left(\frac{x}{2}\right)\right\rangle, \quad \text { if } p \geq 2^{j}-2 N+2,
\end{aligned}
$$

where $\langle\cdot, \cdot\rangle$ is the scalar product of $L^{2}(\mathbb{R})$. The system $\left(x_{j, k}\right)$, where $-N+1 \leq k \leq 2^{j}-$ $N$, is independent. Then we have $\operatorname{dim} X_{j}=2^{j}$ and $X_{j} \cap V_{j}([0,1])=\{0\}$. We remark that $X_{j} \subset v_{j+1}([0,1])$ and $X_{j} \cap V_{j}=\{0\}$. Then $v_{j+1}([0,1])=v_{j}([0,1]) \oplus X_{j}$ (the two spaces have the same dimension) and $X_{j} \subset V_{j+1}$. Since $\operatorname{dim} V_{j}-\operatorname{dim} v_{j}([0,1])$ is increasing and bounded by $\operatorname{dim} V_{j}([0,1])-\operatorname{dim} v_{j}([0,1])=4 N-4$, we conclude that there exists an integer $J$ such that for $j \geq J, V_{j+1}=V_{j} \oplus X_{j}$. Finally, by using Gram-Schmidt method, we get orthonormal wavelet basis on the interval $[0,1]$.

2.2. Biorthogonal multiresolution analyses on the interval. First we give some definitions of biorthogonal multiresolution analysis (denoted by BMRA), then we describe constructions on the interval. 
DEFINITION 2.5. A sequence $\left(V_{j}, V_{j}^{*}\right)$ of closed subspaces of $L^{2}([0,1])$ associated with biorthogonal multiresolution analysis $\left(V_{j}(\mathbb{R}), V_{j}^{*}(\mathbb{R})\right)$ of $L^{2}(\mathbb{R})$ is called a biorthogonal multiresolution analysis of $L^{2}([0,1])$ if

(i) $v_{j}([0,1]) \subset V_{j} \subset V_{j}([0,1])$ and $v_{j}^{*}([0,1]) \subset V_{j}^{*} \subset V_{j}^{*}([0,1])$.

(ii) $V_{j} \subset V_{j+1}$ and $V_{j}^{*} \subset V_{j+1}^{*}$.

(iii) $L^{2}([0,1])=V_{j} \oplus\left(V_{j}^{*}\right)^{\perp}$.

EXAMPLE 2.6 (periodic BMRA). If $\left(V_{j}(\mathbb{R}), V_{j}^{*}(\mathbb{R})\right)$ is a BMRA of $L^{2}(\mathbb{R})$ such that the multiscale functions $g$ and $g^{*}$ have compact support, we can define a BMRA of $L^{2}([0,1])$ associated with $\left(V_{j}(\mathbb{R}), V_{j}^{*}(\mathbb{R})\right)$ by

$$
\begin{aligned}
\tilde{G}_{j, k} & =\sum_{p \in \mathbb{Z}} g_{j, k+p 2^{j}}, \quad \tilde{G}_{j, k}^{*}=\sum_{p \in \mathbb{Z}} g_{j, k+p 2^{j}}^{*}, \\
V_{j} & =\operatorname{Vect}\left\{\tilde{G}_{j, k /[0,1]}, 0 \leq k \leq 2^{j}-1\right\}, \\
V_{j}^{*} & =\operatorname{Vect}\left\{\tilde{G}_{j, k /[0,1]}^{*}, 0 \leq k \leq 2^{j}-1\right\} .
\end{aligned}
$$

It is clear that $\int_{0}^{1} \tilde{G}_{j, k}^{*}(x) \tilde{G}_{j, l}(x) d x=\delta_{k, l}$ for $k, l \in\left\{0, \ldots, 2^{j}-1\right\}$ and $V_{j}, V_{j}^{*}$ are in duality for the scalar product of $L^{2}([0,1])$. This BMRA is adapted to the study of periodic functions.

Let $\left(V_{j}(\mathbb{R}), V_{j}^{*}(\mathbb{R})\right)$ be a BMRA of $L^{2}(\mathbb{R})$ with associated multiscale functions $\left(g, g^{*}\right)$. We assume that supp $g=\left[N_{1}, N_{2}\right]$ and we denote

$$
\begin{aligned}
& P_{i}^{\alpha}(x)=\sum_{k \leq-N_{1}-1} k^{i} g(x-k), \\
& P_{i}^{\beta}(x)=\sum_{k \geq-N_{2}-1} k^{i} g(x-k) .
\end{aligned}
$$

Our construction is based on the following result.

THEOREM 2.7. We consider a BMRA $\left(V_{j}(\mathbb{R}), V_{j}^{*}(\mathbb{R})\right)$ of $L^{2}(\mathbb{R}),\left(g, g^{*}\right)$ are the multiscale functions with compact support and $\left(V_{j}, V_{j}^{*}\right)$ an associated BMRA of $L^{2}([0,1])$. We assume that

(i) $g$ is differentiable and $g^{\prime}(x)=\tilde{g}(x)-\tilde{g}(x-1)$.

(ii) $V_{j}$ contains the functions

$$
\begin{aligned}
& P_{0, j}^{\alpha}(x)=P_{0}^{\alpha}\left(2^{j} x-2^{j}\right)_{/[0,1]}, \\
& P_{0, j}^{\beta}(x)=P_{0}^{\beta}\left(2^{j} x-2^{j}\right)_{/[0,1]} .
\end{aligned}
$$

If we denote

$$
\begin{aligned}
& \tilde{V}_{j}=\left\{f \in L^{2}([0,1]) \mid \exists g \in V_{j}, f=g^{\prime}\right\}, \\
& \tilde{V}_{j}^{*}=\left\{f \in L^{2}([0,1]) \mid f^{\prime} \in V_{j}, \text { and } f(0)=f(1)=0\right\} .
\end{aligned}
$$

Then $\left(\tilde{V}_{j}, \tilde{V}_{j}^{*}\right)$ is a BMRA of $L^{2}([0,1])$. Moreover, if we denote by $P_{j}$ (resp., $\left.\tilde{P}_{j}\right)$ the projector from $L^{2}([0,1])$ into $V_{j}$ (resp., $\left.\tilde{V}_{j}\right)$ parallel to $\left(V_{j}^{*}\right)^{\perp}\left(\right.$ resp., $\left.\left(\tilde{V}_{j}^{*}\right)^{\perp}\right)$, then we 
have the following commutation property

$$
\frac{d}{d x} \circ P_{j}=\tilde{P}_{j} \circ \frac{d}{d x} .
$$

Proof. It is clear that $\tilde{g}(x-k)=\left(\sum_{p=0}^{\infty} g(x-k-p)\right)^{\prime}$ and $\left(\tilde{g}^{*}(x-k)\right)^{\prime}=g^{*}(x-k+$ $1)-g^{*}(x-k)$. Then $\tilde{v}_{j} \subset \tilde{V}_{j}([0,1])$ and $\tilde{v}_{j}^{*} \subset \tilde{V}_{j}^{*}([0,1])$. Moreover, since $V_{j}$ contains the functions $P_{0, j}^{\beta}(x)$, we have $\tilde{V}_{j}([0,1]) \subset \tilde{V}_{j}$ and $\tilde{V}_{j}^{*}([0,1]) \subset \tilde{V}_{j}^{*}$. In the same way, we have $\tilde{V}_{j} \subset \tilde{V}_{j+1}$ and $\tilde{V}_{j}^{*} \subset V_{j+1}^{*}$. To see the duality between $\tilde{V}_{j}$ and $\tilde{V}_{j}^{*}$, we consider a base $\left(\alpha_{0}=1, \alpha_{1}, \ldots, \alpha_{n}\right)$ of $V_{j}$ with $\operatorname{dim} V_{j}=n+1$ and a dual base $\left(\beta_{0}, \beta_{1}, \ldots, \beta_{n}\right)$ of $V_{j}^{*}$. Then the derivation is an isomorphism from $\tilde{V}_{j}^{*}$ onto $\operatorname{Vect}\left(\beta_{1}, \ldots, \beta_{n}\right)$ and from $\operatorname{Vect}\left(\alpha_{1}, \ldots, \alpha_{n}\right)$ onto $\tilde{V}_{j}$. If we define $\tilde{\alpha}_{i}=(d / d x) \alpha_{i}$ and $\tilde{\beta}_{i}=-\int_{0}^{x} \beta_{i}(t) d t$ then by integration, we conclude that the bases $\left(\tilde{\alpha}_{i}\right)$ and $\left(\tilde{\beta}_{i}\right)$ are biorthogonal and we have a duality between $\tilde{V}_{j}$ and $\tilde{V}_{j}^{*}$. Finally the commutation property is satisfied. In fact, we have

$$
\begin{aligned}
\frac{d}{d x} \circ\left(P_{j} f\right) & =\frac{d}{d x}\left\langle f, \beta_{0}\right\rangle 1+\sum_{i=1}^{n}\left\langle f, \beta_{i}\right\rangle \frac{d}{d x} \alpha_{i}=\sum_{i=1}^{n}\left\langle f, \beta_{i}\right\rangle \tilde{\alpha}_{i}, \\
\tilde{P}_{j} \circ\left(\frac{d}{d x} f\right) & =\sum_{i=1}^{n}\left\langle\frac{d}{d x} f, \beta_{i}^{\sim}\right\rangle \tilde{\alpha}_{i}=\sum_{i=1}^{n}\left(\left[f \beta_{i}^{\sim}\right]_{0}^{1}+\left\langle f, \beta_{i}\right\rangle\right) \tilde{\alpha}_{i} \\
& =\sum_{i=1}^{n}\left\langle f, \beta_{i}\right\rangle \tilde{\alpha}_{i} .
\end{aligned}
$$

FUNDAMENTAL EXAMPLE. Let $V_{j}(\mathbb{R})$ be the OMRA of Daubechies where the scale function $\varphi$ is of class $C^{d}$. We denote by $V_{j}^{(d)}(\mathbb{R})$ and $V_{j}^{(-d)}(\mathbb{R})$ the MRA constructed by derivation and integration. Then the theorem described above proves that $V_{j}^{(d)}([0,1])$ and $V_{j}^{(-d)}([0,1]) \cap H_{0}^{d}([0,1])$ form a BMRA of $L^{2}([0,1])$. Moreover, if we denote by $P_{j}^{(d)}$ the projector on $V_{j}^{(d)}([0,1])$ parallel to $\left[V_{j}^{(-d)}([0,1]) \cap H_{0}^{d}([0,1])\right]^{\perp}$, we have

$$
\frac{d}{d x} \circ P_{j}^{(d)}=P_{j}^{(d+1)} \circ \frac{d}{d x} .
$$

We define $g$ and $g^{*}$ by

$$
\left(1-e^{-i \xi}\right)^{d} \hat{g}(\xi)=(i \xi)^{d} \hat{\varphi}(\xi), \quad(i \xi)^{d} \hat{g}^{*}(\xi)=\left(e^{-i \xi}-1\right)^{d} \stackrel{\curlywedge}{\varphi}(\xi) .
$$

The functions $g_{j, k /[0,1]}$ form a basis of $V_{j}^{(-d)}([0,1])$. To construct a basis of $V_{j}^{(-d)}([0,1])$ $\cap H_{0}^{d}([0,1])$ we take the functions $g_{j, k}$ with support in $[0,1]$ and the boundaries functions as follows:

$$
\begin{aligned}
& g_{j, k}^{\alpha *}=\sum_{p=-2 N+2}^{d-1} \alpha_{i, j, p} \mathcal{g}_{j, p /[0,1]}^{*}, \quad 1 \leq i \leq 2 N-2, \\
& g_{j, k}^{\beta *}=\sum_{p=2^{j}-2 N+2}^{2^{j}+d-1} \alpha_{i, j, p} \mathcal{g}_{j, p /[0,1]}^{*}, \quad 1 \leq i \leq 2 N-2 .
\end{aligned}
$$


The real constants $\alpha_{i, j, p}$ satisfy

$$
\int_{0}^{+\infty}\left(\sum_{p=-2 N+2}^{d-1} \alpha_{i, j, p} 2^{j / 2} g^{*}\left(2^{j} x-p\right)\right) 2^{j / 2} g\left(2^{j} x+2 N-1-d-q\right) d x=\delta_{i, q}, \quad 1 \leq i \leq 2 N-2
$$

In this case the following results are proved in [9].

THEOREM 2.8. We assume that $\varphi$ is a $C^{p+\varepsilon}$-function, $p \in \mathbb{N}, p \geq d$, and $\varepsilon>0$. We denote by $P_{j}^{(d)}$ the projector on $V_{j}^{(d)}([0,1])$ parallel to $\left[V_{j}^{(-d)}([0,1]) \cap H_{0}^{d}([0,1])\right]^{\perp}$ and $P_{j}^{(d) *}$ its adjoint. We define $Q_{j}^{(d)}=P_{j}^{(d+1)}-P_{j}^{(d)}, Q_{j}^{(d) *}=P_{j+1}^{(d) *}-P_{j}^{(d) *}$ and $j_{0}$ an integer satisfying $2^{j_{0}}-1 \geq 4 N-4+2 p$. Then

(i) for $f \in L^{2}([0,1]),\|f\|_{2} \approx\left\|P_{j_{0}}^{(d)} f\right\|_{2}+\left(\sum_{j \geq j_{0}}\left\|Q_{j}^{(d)} f\right\|_{2}^{2}\right)^{1 / 2}$.

(ii) for $f \in L^{2}([0,1]),\|f\|_{2} \approx\left\|P_{j_{0}}^{(d) *} f\right\|_{2}+\left(\sum_{j \geq j_{0}}\left\|Q_{j}^{(d) *} f\right\|_{2}^{2}\right)^{1 / 2}$.

(iii) for $k \in \mathbb{Z}$ such that $-d \leq k \leq p-d$, we have

$$
\begin{array}{cc}
f \in H^{k}([0,1]) \Longleftrightarrow P_{j_{0}}^{(d)} f \in L^{2}([0,1]), & \sum_{j \geq j_{0}} 4^{j k}\left\|Q_{j}^{(d)} f\right\|_{2}^{2}<+\infty ; \\
f \in H_{0}^{-k}([0,1]) \Longleftrightarrow P_{j_{0}}^{(d) *} f \in L^{2}([0,1]), & \sum_{j \geq j_{0}} 4^{j k}\left\|Q_{j}^{(d) *} f\right\|_{2}^{2}<+\infty .
\end{array}
$$

REMARK 2.9. The fundamental property $d / d x \circ P_{j}^{(d)}=P_{j}^{(d+1)} \circ d / d x$ allows to study the vector functions with divergence equal to zero (see [9]).

3. Segmented biorthogonal multiresolution analyses and extension operators. We study in this section two constructions of biorthogonal wavelet bases. The first one is based on the OMRA of Daubechies and prove that we can analyze functions in $H^{k}(\mathbb{R})(k \in \mathbb{Z})$ with information in the past $\left.\left.H^{k}(]-\infty, 0\right]\right)(k \in \mathbb{Z})$, the relaxation of the past in the future near zero and information in the future $H_{0}^{k}([0,+\infty))(k \in \mathbb{Z})$. The second construction is based on a symmetric multiresolution analyses with compact support and show how to obtain boundary functions in $[-1,1]$. We obtain, by using extensions, biorthogonal multiresolution analyses of $L^{2}([-1,1])$ from those of $L^{2}([-1,0])$ and $L^{2}([0,1])$.

3.1. General principles of extensions. We begin with some notions of extensions which are important for decompositions of the Sobolev space $H^{k}(\mathbb{R})$. Next we give conditions on extensions to get simple algorithms of wavelet bases.

DEFINITION 3.1. We denote by $\Theta(\mathbb{R})$ the space of continuous operators on $L^{2}(\mathbb{R})$. An operator $A$ of $\Theta(R)$ is called an extension from the interval $I$ into $\mathbb{R}$ if $A$ is a continuous operator from $L^{2}(I)$ into $L^{2}(\mathbb{R})$ such that

(i) $A f_{/ I}=f$.

(ii) For $f$ in $H^{k}(\mathbb{R}),(k \in \mathbb{Z})$, the usual Sobolev space, then $A f \in H^{k}(\mathbb{R})$. 
EXAMPLE 3.2. We consider the interval $I=]-\infty, 0]$ and $f \in C^{k}(I)$ (space of $C^{k}$ functions in $I$ ). We define the operator $A$ by

$$
A f(x)= \begin{cases}f(x), & \text { if } x \leq 0, \\ \sum_{p=1}^{s} \alpha_{p} f\left(\beta_{p} x\right), & \text { if } x>0\end{cases}
$$

where $\beta_{p}$ are real constants such that $\beta_{s}<\beta_{s-1}<\cdots<\beta_{1}<0$. The real constants $\alpha_{p}$ will be chosen such that $A f \in C^{k}(\mathbb{R})$. For this purpose, we must have $\sum_{p=1}^{s} \alpha_{p} \beta_{p}^{m}=1$, $m=0,1, \ldots, k$. The adjoint operator $A^{*}$ of $A$ is defined by

$$
A^{*} g(x)= \begin{cases}g(y)-\sum_{p=1}^{s} \alpha_{p} \beta_{p}^{-1} g\left(\frac{y}{\beta_{p}}\right), & \text { if } y \leq 0, \\ 0, & \text { if } y>0 .\end{cases}
$$

If $g \in C^{k}(\mathbb{R})$ then $A^{*} g \in C^{k}(\mathbb{R})$, since $\sum_{p=1}^{s} \alpha_{p} \beta_{p}^{m-1}=1, m=0,1, \ldots, k$. We conclude that for $s>2 k+2$, the constants $\alpha_{p}$ exist and then $A$ and $A^{*}$ are known.

The biorthogonality of bases depends on the operators $A$ and $A^{*}$. We remark that the operator defined above is not "good" because $A$ or $A^{*}$ does not preserve the property of compact support, if we consider multiresolution analyses with compact support, and special properties near the boundaries [9].

Proposition 3.3. Let $k \in \mathbb{Z}$ and $E$ be an extension from $\left.\left.H^{k}(]-\infty, 0\right]\right)$ into $H^{k}(\mathbb{R})$. Then the operator $f \rightarrow E\left(f_{/]-\infty, 0]}\right)+\left(f-E\left(f_{/]-\infty, 0]}\right)\right)$ constitutes an isomorphism from $H^{k}(\mathbb{R})$ onto $\left.\left.E\left(H^{k}(]-\infty, 0\right]\right)\right) \oplus H_{0}^{k}([0,+\infty[)$.

REMARK 3.4. The extension $E$ relaxes the information of the past, and the function $\left(f-E\left(f_{/]-\infty, 0]}\right)\right)$ is supported by the future axis. We will show how Proposition 3.3 permits to get wavelet bases of $L^{2}(\mathbb{R})$ by using those of $\left.\left.L^{2}(]-\infty, 0\right]\right)$ and $L^{2}([0,+\infty[)$. The main problem is in the definition of extension operators which are adapted to scale, and permit to get regular wavelet bases. This problem is the object of the next section.

3.2. Segmented biorthogonal multiresolution analyses. We introduce now the definition of segmented BMRA.

DEFINITION 3.5. We consider two intervals $I_{1}$ and $I_{2}$ of $\mathbb{R}$ such that $I_{1} \cap I_{2}$ contains one point. Let $\left(V_{j}^{1},\left(V_{j}^{1}\right)^{*}\right)$ be a BMRA of $L^{2}\left(I_{1}\right)$ and $\left(V_{j}^{2},\left(V_{j}^{2}\right)^{*}\right)$ be a BMRA of $L^{2}\left(I_{2}\right)$. We put $I=I_{1} \cup I_{2}$. Then a MRA $\left(V_{j}, V_{j}^{*}\right)$ is called a segmented BMRA of $L^{2}(I)$ if we have $V_{j}=A_{1}\left(V_{j}^{1}\right) \oplus A_{2}\left(V_{j}^{2}\right)$ and $V_{j}^{*}=B_{1}\left(V_{j}^{1}\right)^{*} \oplus B_{2}\left(V_{j}^{2}\right)^{*}$ where $A_{1}, B_{1}$ are two extensions from $L^{2}\left(I_{1}\right)$ into $L^{2}(I)$ and $A_{2}, B_{2}$ are two extensions from $L^{2}\left(I_{2}\right)$ into $L^{2}(I)$.

In the following, we describe two constructions of segmented BMRA. In the first one, we consider $\left.\left.I_{1}=\right]-\infty, 0\right]$ and $I_{2}=[0+\infty$ [ and we get a segmented BMRA of $L^{2}(\mathbb{R})$. In the second construction, we consider $I_{1}=[-1,0]$ and $I_{2}=[0,1]$ and we get a segmented BMRA of $L^{2}([-1,1])$.

3.3. Construction of segmented BMRA of $L^{2}(\mathbb{R})$. The object of this section is to construct a BMRA of $L^{2}(\mathbb{R})$. For this purpose, we consider the OMRA $V_{j}(\mathbb{R})$ of 
Daubechies with associated scaling function $\varphi$ and wavelet $\psi$, and we construct a BMRA of $\left.\left.L^{2}(]-\infty, 0\right]\right)$ and $L^{2}([0+\infty[)$. Next, we use some extension operators to get a BMRA of $L^{2}(\mathbb{R})$.

Recall that the functions $\varphi_{j, k}(x)=2^{j / 2} \varphi\left(2^{j} x-k\right), k \in \mathbb{Z}$, form a Riesz basis of $V_{j}(\mathbb{R})$ and $\psi_{j, k}(x)=2^{j / 2} \psi\left(2^{j} x-k\right), k \in \mathbb{Z}$, form a Riesz basis of $W_{j}(\mathbb{R})$ (orthogonal complement of $V_{j}(\mathbb{R})$ in $\left.V_{j+1}(\mathbb{R})\right)$. We define the extension operators $E_{j}$ and $E_{j}^{\prime}$ by

$$
\begin{aligned}
\left.\left.E_{j}: V_{j}(]-\infty, 0\right]\right) \longrightarrow V_{j}(\mathbb{R}), & \varphi_{j, k /]-\infty, 0]} \longrightarrow \varphi_{j, k}, \\
E_{j}^{\prime}: V_{j}\left(\left[0,+\infty[) \longrightarrow V_{j}(\mathbb{R}),\right.\right. & \varphi_{j, k /[0,+\infty]} \longrightarrow \varphi_{j, k},
\end{aligned}
$$

and the two extension operators $E_{0}^{*}$ and $\left(E_{0}^{*}\right)^{\prime}$

$$
\begin{gathered}
\left.\left.E_{0}^{*}: L^{2}(]-\infty, 0\right]\right) \rightarrow L^{2}(\mathbb{R}), \quad f \rightarrow \bar{f}, \quad \text { where } \bar{f}_{/]-\infty, 0]}=f, \bar{f}_{/] 0,+\infty[}=0, \\
\left(E_{0}^{*}\right)^{\prime}: L^{2}\left(\left[0,+\infty[) \longrightarrow L^{2}(\mathbb{R}), \quad f \rightarrow \bar{f}, \quad \text { where } \bar{f}_{/[0,+\infty[}=f, \bar{f}_{/]-\infty, 0[}=0 .\right.\right.
\end{gathered}
$$

We consider $V_{j}=\operatorname{Vect}\left\{\varphi_{j, k}, k \in \mathbb{Z}\right\}$ (OMRA of Daubechies). To define the dual space, we need to construct the new functions $\varphi_{j, q}^{*}$ given by

$$
\varphi_{j, q}^{*}(x)=\sum_{k=2-2 N}^{-1} a_{j, q, l} \varphi_{j, k /]-\infty, 0]},
$$

where $a_{j, q, l}$ satisfy the following conditions: for $2-2 N \leq k \leq-1$, we have

$$
\int_{-\infty}^{0} \sum_{k=2-2 N}^{-1} a_{j, q, l} 2^{j / 2} \varphi\left(2^{j} x-l\right) \varphi\left(2^{j} x-k\right) d x=\delta_{q, k} .
$$

The precedent system of $(2 N-2)$ equations and $(2 N-2)$ unknowns has one solution because the functions $\varphi_{j, k /]-\infty, 0]}, 2-2 N \leq k \leq-1$, are independent (see [5]). As the conditions on $a_{j, q, l}$ do not depend on $j$, then we have

$$
\varphi_{j, q}^{*}(x)=2^{j / 2} \varphi_{q}^{*}\left(2^{j} x\right)_{/]-\infty, 0]} .
$$

We define now the dual space

$$
V_{j}^{*}=\operatorname{Vect}\left\{\varphi_{j, k}, k \leq 1-2 N, \varphi_{j, k}, k \geq 0 ; 2^{j / 2} \varphi_{q}^{*}\left(2^{j} x\right)_{/]-\infty, 0]}, 2-2 N \leq q \leq-1\right\} .
$$

We remark that $V_{j}^{*}=\operatorname{Vect}\left\{\varphi_{j, k /]-\infty, 0]}\right\} \oplus \operatorname{Vect}\left\{\varphi_{j, k}, \operatorname{supp} \varphi_{j, k} \subset[0,+\infty[\}\right.$ and then $V_{j}^{*} \subset V_{j+1}^{*}$. It is clear that $V_{j}^{*}$ is constructed in order to get a BMRA $\left(V_{j}, V_{j}^{*}\right)$ of $L^{2}(\mathbb{R})$. We consider $W_{j}=V_{j+1} \cap\left(V_{j}^{*}\right)^{\perp}$. To give a basis of $W_{j}$, we need to construct the new wavelets $\alpha_{j, k}$ as follows: for $2-2 N \leq k \leq-N$, we put

$$
\overline{\alpha_{j, k}}=\psi_{j, k}-\sum_{q=2-2 N}^{-1}\left\langle\psi_{j, k}, \varphi_{j, q}^{*}\right\rangle \varphi_{j, q} .
$$

We have $(N-1)$ functions which can be orthonormalized (for the scalar product of $\left.\left.\left.L^{2}(]-\infty, 0\right]\right)\right)$ to get the functions $\alpha_{j, k}, 2-2 N \leq k \leq-N$, indicated above. Then a basis of $W_{j}$ is given by the following functions:

- $\psi_{j, k}, k \leq 1-2 N$. 
- $\alpha_{j, k}, 2-2 N \leq k \leq-N$.

- $\beta_{j+1,2 k}=\varphi_{j+1,2 k}-\sum_{q \geq 0}\left\langle\varphi_{j+1,2 k}, \varphi_{j, q}\right\rangle \varphi_{j, q}-\sum_{q \geq 0}\left\langle\varphi_{j+1,2 k}, \psi_{j, q}\right\rangle \psi_{j, q}, 0 \leq k \leq N-2$.

- $\psi_{j, k}, k \geq 0$.

We construct now a basis of the space $W_{j}^{*}=V_{j+1}^{*} \cap\left(V_{j}\right)^{\perp}$. It is clear that the functions $\alpha_{j, k /]-\infty, 0]}$ are in $V_{j+1}^{*}$. For $2-2 N \leq k \leq-N$, we have

$$
\psi_{j, k}^{*}=\alpha_{j, k /]-\infty, 0]}-\sum_{q=2-2 N}^{-1}\left\langle\alpha_{j, k /]-\infty, 0]}, \varphi_{j, q}^{*}\right\rangle \varphi_{j, q}^{*} .
$$

Obviously, we have $\left\langle\alpha_{j, k}, \psi_{j, q}^{*}\right\rangle_{L^{2}(\mathbb{R})}=\delta_{k, q}$. We denote by $\beta_{j+1,2 k}^{*}, 0 \leq k \leq N-2$, the dual system of $\beta_{j+1,2 k}, 0 \leq k \leq N-2$, for the scalar product of $L^{2}(\mathbb{R})$, and we define

$$
\begin{aligned}
\varphi_{j+1,2 k}^{*}= & \beta_{j+1,2 k}-\sum_{q=2-2 N}^{-1}\left\langle\beta_{j+1,2 k}^{*}, \varphi_{j, q}\right\rangle \varphi_{j, q}^{*} \\
& -\sum_{q=2-2 N}^{-N}\left\langle\beta_{j+1,2 k}^{*}, \psi_{j, q}\right\rangle \psi_{j, q}^{*}-\sum_{q \geq 0}\left\langle\beta_{j+1,2 k}^{*}, \psi_{j, q}\right\rangle \psi_{j, q} .
\end{aligned}
$$

We conclude that a basis of $W_{j}^{*}$ is given by the following functions:

- $\psi_{j, k}, k \leq 1-2 N$.

- $\psi_{j, k}^{*}, 2-2 N \leq k \leq-N$.

- $\varphi_{j+1,2 k}^{*}, 0 \leq k \leq N-2$.

- $\psi_{j, k}, k \geq 0$.

The functions $\varphi_{j, k}, k \in \mathbb{Z}$, form a basis of the space $V_{j}$ and the functions $\varphi_{j, k}^{*}, k \in \mathbb{Z}$, form a basis of the space $V_{j}^{*}$ such that

$$
\begin{gathered}
\operatorname{supp} \varphi_{j, k} \subset\left[\frac{k}{2^{j}}, \frac{k+2 N-1}{2^{j}}\right], \quad \operatorname{supp} \varphi_{j, k}^{*} \subset\left[\frac{k-2 N+2}{2^{j}}, \frac{k+2 N-1}{2^{j}}\right], \\
\left\langle\varphi_{j, k}, \varphi_{j, q}^{*}\right\rangle_{L^{2}(\mathbb{R})}=\delta_{k, q} .
\end{gathered}
$$

The projector $P_{j}$ can be written as

$$
P_{j} f=\sum_{k \in \mathbb{Z}}\left\langle f, \varphi_{j, k}^{*}\right\rangle \varphi_{j, k}
$$

and we have the following property:

$$
P_{j+1} \circ P_{j}=P_{j} \circ P_{j+1}=P_{j} .
$$

The following theorem proves that the MRA $\left(V_{j}, V_{j}^{*}\right)$ described above is a segmented BMRA of $L^{2}(\mathbb{R})$.

THEOREM 3.6. (i) There exist a BMRA $\left.\left.\left.\left.\left(V_{j}(]-\infty, 0\right]\right), V_{j}^{*}(]-\infty, 0\right]\right)\right)$ of $\left.\left.L^{2}(]-\infty, 0\right]\right)$ and a BMRA $\left(V_{j}\left(\left[0+\infty[), V_{j}^{*}\left([0+\infty[))\right.\right.\right.\right.$ of $L^{2}([0+\infty[)$ such that if we denote

$$
\begin{aligned}
V_{j} & \left.\left.=E_{j}\left(V_{j}(]-\infty, 0\right]\right)\right) \oplus E_{j}^{\prime}\left(V_{j}([0+\infty[)),\right. \\
V_{j}^{*} & \left.\left.=E_{0}^{*}\left(V_{j}^{*}(]-\infty, 0\right]\right)\right) \oplus E_{0}^{* \prime}\left(V_{j}^{*}([0+\infty[)),\right.
\end{aligned}
$$

then $\left(V_{j}, V_{j}^{*}\right)$ is a segmented BMRA of $L^{2}(\mathbb{R})$. 
(ii) We consider the spaces

$$
\begin{aligned}
& \left.\left.\left.\left.\left.\left.W_{j}(]-\infty, 0\right]\right)=V_{j+1}(]-\infty, 0\right]\right) \cap\left(V_{j}^{*}(]-\infty, 0\right]\right)\right)^{\perp}, \\
& \left.\left.\left.\left.\left.\left.W_{j}^{*}(]-\infty, 0\right]\right)=V_{j+1}^{*}(]-\infty, 0\right]\right) \cap\left(V_{j}(]-\infty, 0\right]\right)\right)^{\perp} .
\end{aligned}
$$

We define in the same way the spaces $W_{j}\left(\left[0+\infty[) W_{j}^{*}([0+\infty[)\right.\right.$. We denote

$$
\begin{gathered}
\left.\left.W_{j}=E_{j}\left(W_{j}(]-\infty, 0\right]\right)\right) \oplus E_{j}^{\prime}\left(W_{j}([0+\infty[)),\right. \\
\left.\left.W_{j}^{*}=E_{0}^{*}\left(W_{j}^{*}(]-\infty, 0\right]\right)\right) \oplus E_{0}^{* \prime}\left(W_{j}^{*}([0+\infty[)) .\right.
\end{gathered}
$$

Then $W_{j}$ and $W_{j}^{*}$ are in duality for the scalar product of $L^{2}(\mathbb{R})$ and we have $V_{j+1}=$ $V_{j} \oplus W_{j}$ and $V_{j+1}^{*}=V_{j}^{*} \oplus W_{j}^{*}$. If we denote

$$
\begin{aligned}
\left.\left.V_{j}^{(1)}(]-\infty, 0\right]\right) & \left.\left.\left.\left.=\left\{f \in L^{2}(]-\infty, 0\right]\right) / \exists g \in V_{j}(]-\infty, 0\right]\right), f=g^{\prime}\right\}, \\
\left.\left.V_{j}^{(-1)}(]-\infty, 0\right]\right) & \left.\left.\left.\left.=\left\{f \in L^{2}(]-\infty, 0\right]\right) / f^{\prime} \in V_{j}^{*}(]-\infty, 0\right]\right), \text { and } f(0)=0\right\},
\end{aligned}
$$

then $\left.\left.\left.\left.\left(V_{j}^{(1)}(]-\infty, 0\right]\right), V_{j}^{(-1)}(]-\infty, 0\right]\right)\right)$ is a BMRA of $\left.\left.L^{2}(]-\infty, 0\right]\right)$. There exists also a BMRA of $L^{2}\left(\left[0+\infty[)\right.\right.$ denoted by $\left(V_{j}^{(1)}\left(\left[0,+\infty[), V_{j}^{(-1)}([0,+\infty[))\right.\right.\right.$.

(iii) If we define $V_{j}^{(1)}$ and $V_{j}^{(-1)}$ in the same way as (3.15) by replacing $\left.\left.V_{j}(]-\infty, 0\right]\right)$ by $\left.\left.V_{j}^{(1)}(]-\infty, 0\right]\right)$ and $\left.\left.V_{j}^{*}(]-\infty, 0\right]\right)$ by $\left.\left.V_{j}^{(-1)}(]-\infty, 0\right]\right)$ (also for the spaces defined on $\left[0,+\infty[)\right.$, then $\left(V_{j}^{(1)}, V_{j}^{(-1)}\right)$ is a BMRA of $L^{2}(\mathbb{R})$. Moreover, if $P_{j}$ is the projector from $L^{2}(\mathbb{R})$ into $V_{j}$ parallel to $\left(V_{j}^{*}\right)^{\perp}$ and $P_{j}^{(1)}$ is the projector from $L^{2}(\mathbb{R})$ into $V_{j}^{(1)}$ parallel to $\left(V_{j}^{(-1)}\right)^{\perp}$, then we have

$$
\frac{d}{d x} \circ P_{j}=P_{j}^{(1)} \circ \frac{d}{d x} .
$$

Proof. We consider the BMRA $\left(V_{j}, V_{j}^{*}\right)$ of $L^{2}(\mathbb{R})$ described above. In the following we prove the properties (i) and (ii). By taking the restrictions, respectively, on ] $-\infty, 0]$ and $\left[0,+\infty\right.$ [ of functions of $V_{j}$ and $V_{j}^{*}$. We get the spaces

$$
\begin{aligned}
\left.\left.V_{j}(]-\infty, 0\right]\right) & =\operatorname{Vect}\left\{\varphi_{j, k /]-\infty, 0]}, k \leq-1\right\}, \\
\left.\left.V_{j}^{*}(]-\infty, 0\right]\right) & =\operatorname{Vect}\left\{\varphi_{j, k}, k \leq 1-2 N, \varphi_{j, k}^{*}, 2-2 N \leq k \leq-1\right\}, \\
V_{j}([0+\infty[) & =\operatorname{Vect}\left\{\varphi_{j, k}, k \geq 0\right\}, \\
V_{j}^{*}([0+\infty[) & =\operatorname{Vect}\left\{\varphi_{j, k}, k \geq 0\right\} .
\end{aligned}
$$

In the same way, the spaces defined in (3.16) are completely described. In fact, we have

$$
\begin{aligned}
\left.\left.W_{j}(]-\infty, 0\right]\right) & =\operatorname{Vect}\left\{\psi_{j, k}, k \leq 1-2 N ; \alpha_{j, k /]-\infty, 0]}, 2-2 N \leq k \leq-N\right\}, \\
\left.\left.W_{j}^{*}(]-\infty, 0\right]\right) & =\operatorname{Vect}\left\{\psi_{j, k}, k \leq 1-2 N, \psi_{j, k}^{*}, 2-2 N \leq k \leq-N\right\}, \\
W_{j}([0,+\infty[) & =\operatorname{Vect}\left\{\beta_{j+1,2 k}, 0 \leq k \leq N-2, \psi_{j, k}, k \geq 0\right\}, \\
W_{j}^{*}([0,+\infty[) & =\operatorname{Vect}\left\{\varphi_{j+1,2 k /[0+\infty[}^{*}, 0 \leq k \leq N-2, \psi_{j, k}, k \geq 0\right\} .
\end{aligned}
$$


Then the properties (3.15) and (3.16) are proved. To prove (3.18), we denote

$$
\begin{aligned}
\frac{d}{d x} \varphi_{j, k}(x) & =2^{j}\left(\varphi_{j, k}^{(1)}(x)-\varphi_{j+1, k}^{(1)}(x)\right), \\
\varphi_{j, k}^{*(-1)}(x) & =2^{-j} \int_{-\infty}^{x}\left(\varphi_{j, k-1}^{*}(t)-\varphi_{j, k}^{*}(t)\right) d t .
\end{aligned}
$$

To show that $\varphi_{j, k}^{*(-1)}$ has compact support, we prove that $\int_{-\infty}^{+\infty} \varphi_{j, k}^{*}(x) d x=2^{-j / 2}$ (because the functions $\varphi_{j, k}^{*}$ have compact support). In fact, we have

$$
1=2^{j / 2} \sum_{q \in \mathbb{Z}} \varphi_{j, q}(x)
$$

we then get

$$
\begin{aligned}
\int_{-\infty}^{+\infty} \varphi_{j, k}^{*}(x) d x & =\int_{-\infty}^{+\infty} \varphi_{j, k}^{*}(x) 1 d x \\
& =\int_{-\infty}^{+\infty} \varphi_{j, k}^{*}(x)\left(2^{j / 2} \sum_{q \in \mathbb{Z}} \varphi_{j, q}(x)\right) d x \\
& =\int_{-\infty}^{+\infty} 2^{j / 2} \varphi_{j, q}(x) \varphi_{j, k}^{*}(x) d x \\
& =2^{-j / 2}
\end{aligned}
$$

We describe in the same way as (3.16) the spaces defined in (3.18) and (3.19). The commutation property is proved in [9, Proposition 2.5]. Then, Theorem 3.6 is completely proved.

We remark that $\left.\left.\operatorname{supp} \varphi_{j, k}^{*} \subset\right]-\infty, 0\right]$ for $k \leq-1$ and $\operatorname{supp} \varphi_{j, k}^{*} \subset[0,+\infty[$ for $k \geq 0$, then we have two projectors

$$
P_{j}^{-} f=\sum_{k \leq-1}\left\langle f, \varphi_{j, k}^{*}\right\rangle \varphi_{j, k}
$$

the projector in the past which is relaxed in the future, and

$$
P_{j}^{+} f=\sum_{k \geq 0}\left\langle f, \varphi_{j, k}^{*}\right\rangle \varphi_{j, k},
$$

the projector in the future.

We have similar properties as (3.25) and (3.26) for the projector $Q_{j}=P_{j+1}-P_{j}$, because the associated wavelets satisfy the same properties as the scaling functions $\varphi_{j, k}^{*}$.

The space $V_{j}^{(1)}$ is equal to $V_{j}^{(1)}(\mathbb{R})$ (the MRA constructed by one derivation on $V_{j}(\mathbb{R})$ ) with a basis $\varphi_{j, k}^{(1)}, k \in \mathbb{Z}$, and the space $V_{j}^{(-1)}$ has a basis $\varphi_{j, k}^{*(-1)}, k \in \mathbb{Z}$, such that

$$
\begin{gathered}
\left\langle\varphi_{j, k}, \varphi_{j, q}^{*}\right\rangle_{L^{2}(\mathbb{R})}=\delta_{k, q}, \\
\left.\left.\operatorname{supp} \varphi_{j, k}^{*(-1)} \subset\right]-\infty, 0\right], \quad \text { for } k \leq 0, \\
\operatorname{supp} \varphi_{j, k}^{*(-1)} \subset[0,+\infty[, \quad \text { for } k \geq 0 .
\end{gathered}
$$


The projector $P_{j}^{(1)}$ is given by

$$
P_{j}^{(1)} f=\sum_{k \in \mathbb{Z}}\left\langle f, \varphi_{j, k}^{*(-1)}\right\rangle \varphi_{j, k}^{(1)},
$$

and we have the property

$$
P_{j+1}^{(1)} \circ P_{j}^{(1)}=P_{j}^{(1)} \circ P_{j+1}^{(1)}=P_{j}^{(1)} .
$$

The associated wavelets may be constructed in the same way. In fact we denote

$$
\psi_{j, k}^{(1)}(x)=\frac{d}{d x} \psi_{j, k}(x), \quad \psi_{j, k}^{*(-1)}(x)=2^{j} \int_{-\infty}^{x} \psi_{j, k}^{*}(t) d t .
$$

We can generalize the result described above. In fact, the same extensions give the following result.

COROLLARY 3.7. There exist a BMRA $\left.\left.\left.\left.\left(V_{j}^{(d)}(]-\infty, 0\right]\right), V_{j}^{(-d)}(]-\infty, 0\right]\right)\right)$ of $\left.\left.L^{2}(]-\infty, 0\right]\right)$ and a BMRA $\left(V_{j}^{(d)}\left(\left[0+\infty[), V_{j}^{(-d)}\left([0+\infty[))\right.\right.\right.\right.$ of $L^{2}([0+\infty[)$ such that if we denote

$$
\begin{aligned}
V_{j}^{(d)} & \left.\left.=E_{j}\left(V_{j}^{(d)}(]-\infty, 0\right]\right)\right) \oplus E_{j}^{\prime}\left(V_{j}^{(d)}([0+\infty[)),\right. \\
V_{j}^{(-d)} & \left.\left.=E_{0}^{*}\left(V_{j}^{(-d)}(]-\infty, 0\right]\right)\right) \oplus E_{0}^{* \prime}\left(V_{j}^{(-d)}([0+\infty[)) .\right.
\end{aligned}
$$

Then $\left(V_{j}^{(d)}, V_{j}^{(-d)}\right)$ is a segmented BMRA of $L^{2}(\mathbb{R})$.

If $P_{j}^{(d)}$ is the projector from $L^{2}(\mathbb{R})$ into $V_{j}^{(d)}$ parallel to $\left(V_{j}^{(-d)}\right)^{\perp}$ and $P_{j}^{(d+1)}$ is the projector from $L^{2}(\mathbb{R})$ into $V_{j}^{(d+1)}$ parallel to $\left(V_{j}^{-(d+1)}\right)^{\perp}$, then we have

$$
\frac{d}{d x} \circ P_{j}^{(d)}=P_{j}^{(d+1)} \circ \frac{d}{d x} .
$$

REMARK 3.8. The extensions $E_{j}$ and $E_{j}^{\prime}$ expressed in (3.3) permit to require the same regularity and localization of basic functions. If we assume that $\varphi$ is a $C^{p+\varepsilon}$. function then, the BMRA $\left.\left.\left.\left.V_{j}^{(d)}(]-\infty, 0\right]\right), V_{j}^{(-d)}(]-\infty, 0\right]\right)$ is adapted to the study of the Sobolev spaces $\left.\left.H^{k}(]-\infty, 0\right]\right)$ and $\left.\left.H_{0}^{k}(]-\infty, 0\right]\right)$ for $0 \leq k \leq p-d\left(\right.$ or $\left.\left.H^{-k}(]-\infty, 0\right]\right)$ and $\left.\left.H_{0}^{-k}(]-\infty, 0\right]\right)$ for $\left.0 \leq k \leq d\right)$. We have the same result for the BMRA $V_{j}^{(d)}([0,+\infty[)$, $V_{j}^{(-d)}\left(\left[0+\infty[)\right.\right.$ of $L^{2}([0,+\infty[)$.

As a consequence, the BMRA $\left(V_{j}^{(d)}, V_{j}^{(-d)}\right)$ of $L^{2}(\mathbb{R})$ is adapted to the study of the Sobolev spaces $H^{k}(\mathbb{R})$ for $0 \leq k \leq p-d$ and $H^{-k}(\mathbb{R})$ for $0 \leq k \leq d$.

3.4. Construction of segmented BMRA of $L^{2}([-1,1])$. We consider the functions $\varphi$ and $\varphi^{*}$ defined by

$$
\begin{aligned}
\hat{\varphi}(\xi) & =\prod_{j=1}^{\infty} m\left(\frac{\xi}{2^{j}}\right), \quad \text { with } m(\xi)=\frac{1+\cos \xi}{2}, \\
\hat{\varphi}^{*}(\xi) & =\prod_{j=1}^{\infty} m^{*}\left(\frac{\xi}{2^{j}}\right), \quad \text { with } m^{*}(\xi)=\left(\frac{1+\cos \xi}{2}\right)(2-\cos \xi),
\end{aligned}
$$

where “人” is the classical Fourier transform on $\mathbb{R}$. 
These multiscales define a BMRA of $L^{2}(\mathbb{R})$. We denote by $U_{j}(\mathbb{R})$ the closed linear hull of $\varphi_{j, k}, k \in \mathbb{Z}$, where $\varphi_{j, k}(x)=2^{j / 2} \varphi\left(2^{j} x-k\right)$, and in the same way $U_{j}^{*}(\mathbb{R})$ the closed linear hull of $\varphi_{j, k}^{*}, k \in \mathbb{Z}$, where $\varphi_{j, k}^{*}(x)=2^{j / 2} \varphi^{*}\left(2^{j} x-k\right)$. The important properties of $\varphi$ and $\varphi^{*}$ are

$$
\varphi(x)=(1-|x|)^{+} .
$$

(We remark that $\varphi$ is a spline function and $U_{j}(\mathbb{R})$ is the space of continued functions on $\mathbb{R}$ which are affine in every diadic interval $\left[k / 2^{j},(k+1) / 2^{j}\right]$.)

$$
\varphi \text { and } \varphi^{*} \text { are in } L^{2}(\mathbb{R}),
$$

There exists $\varepsilon>0$ such that $\varphi \in H^{1+\varepsilon}(\mathbb{R})$,

$$
\begin{gathered}
\operatorname{supp} \varphi=[-1,1], \quad \varphi(x)=\varphi(-x), \\
\sum_{k \in \mathbb{Z}} \varphi(x-k)=1, \quad \sum_{k \in \mathbb{Z}} k \varphi(x-k)=x, \\
\operatorname{supp} \varphi^{*}=[-2,2], \quad \varphi^{*}(x)=\varphi *(-x), \\
\sum_{k \in \mathbb{Z}} \varphi^{*}(x-k)=1, \quad \sum_{k \in \mathbb{Z}} k \varphi^{*}(x-k)=x, \\
\varphi(x)=\frac{1}{2} \varphi(2 x+1)+\varphi(2 x)+\frac{1}{2} \varphi(2 x-1), \\
\varphi^{*}(x)=\frac{1}{2} \varphi *(2 x+2)+\frac{1}{2} \varphi *(2 x+1)+\frac{3}{2} \varphi^{*}(2 x) \\
+\frac{1}{2} \varphi^{*}(2 x-1)-\frac{1}{4} \varphi *(2 x-2) .
\end{gathered}
$$

(We see that $U_{j}(\mathbb{R}) \subset U_{j+1}(\mathbb{R})$ and $U_{j}^{*}(\mathbb{R}) \subset U *_{j+1}(\mathbb{R})$.)

$$
\left\langle\varphi_{j, k}, \varphi_{j, q}^{*}\right\rangle_{L^{2}(\mathbb{R})}=\delta_{k, q} .
$$

The last point allows that the functions $\varphi_{j, k}, k \in \mathbb{Z}$, form a Riesz base of $V_{j}(\mathbb{R})=$ $U_{j}(\mathbb{R}) \cap L^{2}(\mathbb{R})$ and $\varphi_{j, k}^{*}, k \in \mathbb{Z}$, form a Riesz base of $V_{j}^{*}(\mathbb{R})=U_{j}^{*}(\mathbb{R}) \cap L^{2}(\mathbb{R})$ such that $L^{2}(\mathbb{R})=V_{j}(\mathbb{R}) \oplus\left(V_{j}^{*}(\mathbb{R})\right)^{\perp}$.

The projector $P_{j}$ from $L^{2}(\mathbb{R})$ into $V_{j}$ parallel to $\left(V_{j}^{*}\right)^{\perp}$ can be written in the form

$$
P_{j} f=\sum_{k \in \mathbb{Z}}\left\langle f, \varphi_{j, k}^{*}\right\rangle \varphi_{j, k},
$$

and satisfies $P_{j+1} \circ P_{j}=P_{j} \circ P_{j+1}=P_{j}$, such that $Q_{j}=P_{j+1}-P_{j}$ is a projector from $L^{2}(\mathbb{R})$ into $W_{j}(\mathbb{R})=\operatorname{Im} Q_{j}=V_{j+1}(\mathbb{R}) \oplus\left(V_{j}(\mathbb{R})\right)^{\perp}$ parallel to $\left(W_{j}^{*}(\mathbb{R})\right)^{\perp}$, where $W_{j}^{*}(\mathbb{R})=$ $\left(\operatorname{Ker} Q_{j}\right)^{\perp}=V_{j+1}^{*}(\mathbb{R}) \oplus\left(V_{j}(\mathbb{R})\right)^{\perp}$. A base of $W_{j}(\mathbb{R})$ is given by the functions $\psi_{j, k}(x)=$ $2^{j / 2} \psi\left(2^{j} x-k\right), k \in \mathbb{Z}$, where $\hat{\psi}(2 \xi)=e^{-i \xi} \overline{m(\xi+\pi)} \hat{\varphi}(\xi)$. In the same way, a base of $W_{j}^{*}(\mathbb{R})$ is given by the functions $\psi_{j, k}^{*}(x)=2^{j / 2} \psi^{*}\left(2^{j} x-k\right), k \in \mathbb{Z}$, where $\widehat{\psi^{*}}(2 \xi)=$ $e^{-i \xi} \overline{m(\xi+\pi)} \widehat{\varphi^{*}}(\xi)$. Moreover, the two bases are in duality for the scalar product of $L^{2}(\mathbb{R}):$

$$
\left\langle\psi(x), \psi^{*}(x-k)\right\rangle_{L^{2}(\mathbb{R})}=\delta_{k, 0}, \quad Q_{j} f=\sum_{k \in \mathbb{Z}}\left\langle f, \psi_{j, k}^{*}\right\rangle \psi_{j, k}
$$


The fundamental properties of $\psi$ and $\psi^{*}$ are

$$
\begin{gathered}
\operatorname{supp} \psi=[-1,2], \quad \psi(x)=\psi(1-x), \\
\int_{-\infty}^{+\infty} \psi(x) d x=\int_{-\infty}^{+\infty} x \psi(x) d x=0, \\
\left\langle\psi(x), \varphi^{*}(x-k)\right\rangle_{L^{2}(\mathbb{R})}=0 \quad \text { for } k \in \mathbb{Z}, \\
\operatorname{supp} \psi^{*}=[-1,2], \quad \psi^{*}(x)=\psi^{*}(1-x), \\
\int_{-\infty}^{+\infty} \psi^{*}(x) d x=\int_{-\infty}^{+\infty} x \psi^{*}(x) d x=0, \\
\left\langle\psi^{*}(x), \varphi(x-k)\right\rangle_{L^{2}(\mathbb{R})}=0 \quad \text { for } k \in \mathbb{Z}, \\
\left\langle\psi_{j, k}, \psi_{j, q}^{*}\right\rangle_{L^{2}(\mathbb{R})}=\delta_{j, j^{\prime}} \delta_{k, k^{\prime}} .
\end{gathered}
$$

We construct now a segmented BMRA of $L^{2}([-1,1])$ by using those in $L^{2}([-1,0])$ and $L^{2}([0,1])$ and the extension operators $E_{j}$ and $E_{j}^{\prime}$ described above.

We define the following spaces:

$$
\begin{aligned}
V_{j}([-1,0]) & =\operatorname{Vect}\left\{\varphi_{j}^{\alpha}=\sum_{k \leq 2-2^{j}} \varphi_{j, k /[-1,0]}, \varphi_{j, k}, 3-2^{j} \leq k \leq-3, \varphi_{j}^{\beta}=\sum_{k=-2}^{2} \varphi_{j, k /[-1,0]}\right\}, \\
V_{j}^{*}([-1,0]) & =\operatorname{Vect}\left\{\varphi_{j, k}^{*}, 2-2^{j} \leq k \leq-2\right\}, \\
V_{j}([0,1]) & =\operatorname{Vect}\left\{\varphi_{j, k}, 3 \leq k \leq 2^{j}-3, \varphi_{j}^{\lambda}=\sum_{k \geq 2^{j}-2} \varphi_{j, k /[0,1]}\right\}, \\
V_{j}^{*}([0,1]) & =\operatorname{Vect}\left\{\varphi_{j, k}^{*}, 3 \leq k \leq 2^{j}-2\right\} .
\end{aligned}
$$

It is clear that $V_{j}([-1,0])$ and $V_{j}^{*}([-1,0])$ are in duality because $\operatorname{supp} \varphi_{j, k}^{*} \subset[-1,0]$ for $2-2^{j} \leq k \leq-2$. We have the same result for $V_{j}([0,1])$ and $V_{j}^{*}([0,1])$. We apply extensions as follows:

- The function $\varphi_{j}^{\beta}$ is extended by $\widetilde{\varphi_{j}^{\beta}}=\sum_{k=-2}^{2} \varphi_{j, k}$.

- The functions $\varphi_{j, k}^{*}, 2-2^{j} \leq k \leq-2$ and $3 \leq k \leq 2^{j}-2$, are extended by zero.

We get a BMRA of $L^{2}([-1,1])$, which is symmetric and with compact support where

$V_{j}([-1,1])=\operatorname{Vect}\left\{\varphi_{j}^{\alpha}, \varphi_{j, k}, 3-2^{j} \leq k \leq-3, \widetilde{\varphi_{j}^{\beta}}, \varphi_{j, k}, 3 \leq k \leq 2^{j}-3, \varphi_{j}^{\lambda}\right\}$,

$V_{j}^{*}([-1,1])=\operatorname{Vect}\left\{\varphi_{j, k}^{*}, 2-2^{j} \leq k \leq-2, \varphi_{j, k}^{*}, 3 \leq k \leq 2^{j}-2\right\}$.

In the same way, we get the associated wavelet spaces $W_{j}([-1,1])$ and $W_{j}^{*}([-1,1])$.

Proposition 3.9. If $f$ is a function of $L^{2}([-1,1])$, then

$$
f \in H^{1}([-1,1]) \Longleftrightarrow\left\|P_{j} f\right\|_{2}+\left(\sum_{j \geq j_{0}} 4^{j}\left\|Q_{j} f\right\|_{2}^{2}\right)^{1 / 2}<+\infty .
$$


Proof. If $f \in H^{1}([-1,1]),\|f\|_{H^{1}}$ is calculated by $\|f\|_{2}+\left\|f^{\prime}\right\|_{2}$. We have

$$
f=P_{j_{0}} f+\sum_{j \geq j_{0}} Q_{j} f, \quad\|f\|_{2}=\left\|P_{j_{0}} f\right\|_{2}+\left(\sum_{j \geq j_{0}}\left\|Q_{j} f\right\|_{2}^{2}\right)^{1 / 2},
$$

where $Q_{j} f$ is given by

$$
Q_{j} f=\sum_{k \in \mathbb{Z}}\left\langle f, \psi_{j, k}^{*}\right\rangle \psi_{j, k}=\sum_{k \in \mathbb{Z}} 2^{-j}\left\langle f^{\prime}, \eta_{j, k}^{*}\right\rangle \psi_{j, k},
$$

and $\eta^{*}(x)=-\int_{-1}^{x} \psi^{*}(t) d t$. The functions 1 and $x$ belong to $V_{j}([-1,1])$ (see (3.36)), then we get $\int_{-1}^{1} x^{\alpha} \eta^{*}(x) d x=0$, for $0 \leq \alpha \leq 1$ (see [8]). We have

$$
4^{j}\left\|Q_{j} f\right\|_{2}^{2}=\left\langle\sum_{k \in \mathbb{Z}}\left\langle f^{\prime}, \eta_{j, k}^{*}\right\rangle \psi_{j, k}, \sum_{p \in \mathbb{Z}}\left\langle f^{\prime}, \eta_{j, p}^{*}\right\rangle \psi_{j, p}\right\rangle,
$$

and for a sequence $\left(\lambda_{j, k}\right)_{j, k} \in \mathbb{Z}^{2}$, we have

$$
\begin{aligned}
\sum_{j \in \mathbb{Z}} \sum_{k \in \mathbb{Z}}\left\langle f^{\prime}, \eta_{j, k}^{*}\right\rangle \lambda_{j, k} & =\left\langle f^{\prime}, \sum_{j \in \mathbb{Z}} \sum_{k \in \mathbb{Z}} \lambda_{j, k} \eta_{j, k}^{*}\right\rangle \\
& \leq\left\|f^{\prime}\right\|_{2}\left\|\sum_{j \in \mathbb{Z}} \sum_{k \in \mathbb{Z}} \lambda_{j, k} \eta_{j, k}^{*}\right\|_{2} \\
& \leq C\left\|f^{\prime}\right\|_{2}\left(\sum_{j \in \mathbb{Z}} \sum_{k \in \mathbb{Z}}\left|\lambda_{j, k}\right|^{2}\right)^{1 / 2},
\end{aligned}
$$

where $C$ is a positive constant. Then we get $\sum_{j \geq j_{0}} 4^{j}\left\|Q_{j} f\right\|_{2}^{2} \leq C^{\prime}\left\|f^{\prime}\right\|_{2}$, where $C^{\prime}$ is a positive constant. To prove the other inequalities, we write

$$
f=P_{j_{0}} f+\sum_{j \geq j_{0}} Q_{j} f
$$

then we have

$$
\frac{d}{d x} f=\frac{d}{d x} P_{j_{0}} f+\sum_{j \geq j_{0}} \frac{d}{d x} Q_{j} f, \quad\left\|\frac{d}{d x} P_{j_{0}} f\right\|_{2} \leq C\left\|P_{j_{0}} f\right\|_{2},
$$

where $C$ is a positive constant. To see that $f \in H^{1}([-1,1])$, we must estimate $\| \sum_{j \geq j_{0}}(d /$ $d x) Q_{j} f \|_{2}$. We have

$$
\frac{d}{d x} Q_{j} f=\sum_{k \in \mathbb{Z}} 2^{j}\left\langle f, \eta_{j, k}^{*}\right\rangle\left(\frac{d}{d x} \psi_{j, k}\right)=\sum_{k \in \mathbb{Z}}\left\langle\frac{d}{d x} f, \eta_{j, k}^{*}\right\rangle\left(\frac{d}{d x} \psi_{j, k}\right),
$$

we then get

$$
\left\|\frac{d}{d x} Q_{j} f\right\|_{2} \cong \sum_{k \in \mathbb{Z}}\left|\left\langle\frac{d}{d x} f, \eta_{j, k}^{*}\right\rangle\right|^{2} \cong 4^{j}\left\|Q_{j} f\right\|_{2}^{2} .
$$

The properties (3.45) and (3.52) give the result.

REMARK 3.10. We characterize in the same way the Sobolev space $H_{0}^{1}([-1,1])$ because it is sufficient to replace the projector operator $P_{j}$ (resp., $Q_{j}$ ) by $P_{j}^{*}$ (resp., by 
$\left.Q_{j}^{*}\right)$. We prove then that $\|f\|_{H^{1}}$ and $\left\|P_{j}^{*} f\right\|_{2}+\left(\sum_{j \geq j_{0}} 4^{j}\left\|Q_{j}^{*} f\right\|_{2}^{2}\right)^{1 / 2}$ are equivalent in $H_{0}^{1}([-1,1])$.

Conclusion. We have constructed in this paper two multiresolution analyses (OMRA and BMRA) of $L^{2}([0,1])$ which are generated by a finite number of basis functions. For the first one, we used a direct method based on the result described in [9] to define an orthonormal multiresolution analysis on $[0,1]$ which is regular and has compact support. For the second one, we used the idea of "derivation and integration" to get new biorthogonal multiresolution analyses on the interval. In this case, we get the commutation property (2.13) between scale projectors and differentiability. Next, we use the decomposition method to construct two segmented biorthogonal multiresolution analyses. For the first one, we show that if we consider an extension operator $E$ from $\left.\left.H^{k}(]-\infty, 0\right]\right)$ into $H^{k}(\mathbb{R})$, we get decomposition of the Sobolev space $H^{k}(\mathbb{R})$ $(k \in \mathbb{Z})$ by using an isomorphism between the space $H^{k}(\mathbb{R})(k \in \mathbb{Z})$ and the space $\left.\left.E\left(H^{k}(]-\infty, 0\right]\right)\right)+H_{0}^{k}([0,+\infty[)$. This isomorphism permit to get biorthogonal multiresolution analyses of $H^{k}(\mathbb{R})(k \in \mathbb{Z})$ based on those of $\left.\left.L^{2}(]-\infty, 0\right]\right)$ and $L^{2}([0,+\infty[)$. These multiscale analyses satisfy the commutation property between scale projectors and derivation. All segmented BMRA constructed in this case prove that we can analyze functions in $H^{k}(\mathbb{R})(k \in \mathbb{Z})$ with information in the past $\left.\left.H^{k}(]-\infty, 0\right]\right)$ $(k \in \mathbb{Z})$, the relaxation of the past in the future near zero and information in the future $H_{0}^{k}([0,+\infty[)(k \in \mathbb{Z})$. To prove the second one, we have constructed in the first time orthogonal wavelet bases in the spaces $L^{2}([-1,0])$ and $L^{2}([0,1])$. Using extensions, we get multiresolution analysis on the interval $[-1,1]$. These bases are associated to simple algorithms and are adapted to the study of the Sobolev spaces $H^{1}([-1,1])$ and $H_{0}^{1}([-1,1])$.

Recall that all bases constructed in this work satisfy the lifting scheme [10, 14]; thus, we get wavelet bases with compact support and with the same regularity as for Daubechies bases.

\section{REFERENCES}

[1] Z. Ciesielski and T. Figiel, Spline bases in classical function spaces on compact $C^{\infty}$ manifolds. II, Studia Math. 76 (1983), no. 2, 96-136. MR 85f:46060b. Zbl 0599.46042.

[2] __ Spline bases in classical function spaces on compact $C^{\infty}$ manifolds. I, Studia Math. 76 (1983), no. 1, 1-58. MR 85f:46060a. Zbl 0599.46041.

[3] A. Cohen, W. Dahmen, and R. DeVore, Multiscale decompositions on bounded domains, Trans. Amer. Math. Soc. 352 (2000), no. 8, 3651-3685. MR 2000m:42025. Zbl 0945.42018.

[4] W. Dahmen and R. Schneider, Composite wavelet bases, IGPM 133, RWTH, Aachen, 1995.

[5] Wavelets on manifolds. I. Construction and domain decomposition, SIAM J. Math. Anal. 31 (1999), no. 1, 184-230. MR 2000k:65242. Zbl 0955.42025.

[6] I. Daubechies, Orthonormal bases of wavelets with compact support, Comm. Pure Appl. Math. 42 (1988), 906-996.

[7] A. Jouini, Constructions de bases d'ondelettes sur les variétés, Ph.D. thesis, Orsay, 1993.

[8] A. Jouini and P. G. Lemarie-Rieusset, Ondelettes sur un ouvert borné du plan, preprint, 1992.

[9] _ Analyse multi-résolution bi-orthogonale sur l'intervalle et applications [Biorthogonal multiresolution analysis on the interval, and applications], Ann. Inst. H. Poincaré Anal. Non Linéaire 10 (1993), no. 4, 453-476 (French). MR 95f:42048. 
[10] P. G. Lemarie-Rieusset, Analyses multi-résolutions non orthogonales, commutation entre projecteurs et dérivation et ondelettes vecteurs à divergence nulle [Nonorthogonal multiresolution analyses, commutation between projectors and differentiation and divergence-free vector wavelets], Rev. Mat. Iberoamericana 8 (1992), no. 2, 221-237 (French). MR 94d:42044. Zbl 0874.42022.

[11] R. Masson, Méthodes d'ondelettes en simulation numérique pour les problèmes elliptiques et point selle, Ph.D. thesis, Université Paris VI, 1999.

[12] Y. Meyer, Ondelettes sur l'intervalle [Wavelets on the interval], Rev. Mat. Iberoamericana 7 (1991), no. 2, 115-133 (French). MR 92m:42001. Zbl 0753.42015.

[13] E. M. Stein, Singular Integrals and Differentiability Properties of Functions, Princeton Mathematical Series, no. 30, Princeton University Press, New Jersey, 1970. MR 44\#7280. Zbl 0207.13501.

[14] W. Sweldens, The lifting scheme: a custom-design construction of biorthogonal wavelets, Appl. Comput. Harmon. Anal. 3 (1996), no. 2, 186-200. MR 97b:42060. Zbl 0874.65104 .

Abdellatif Jouini: Department of Mathematics, Faculty of SCiENCES OF TUNis, CAMPus 1060 TUNIS, TUNISIA

E-mail address: abde11atif.jouini@fst.rnu.tn

Khalifa Trimèche: Department of Mathematics, Faculty of SCiences of Tunis, CamPUS 1060 TUNIS, TUNISIA

E-mail address: kha1 ifa.trimeche@fst.rnu.tn 


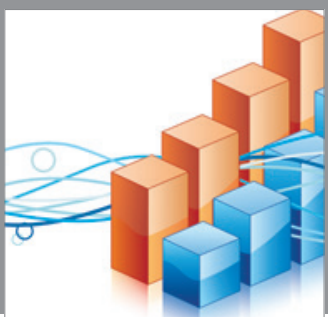

Advances in

Operations Research

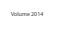

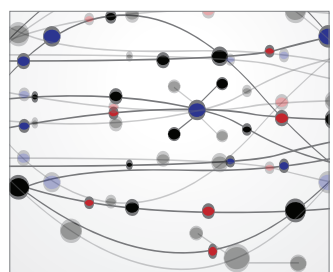

\section{The Scientific} World Journal
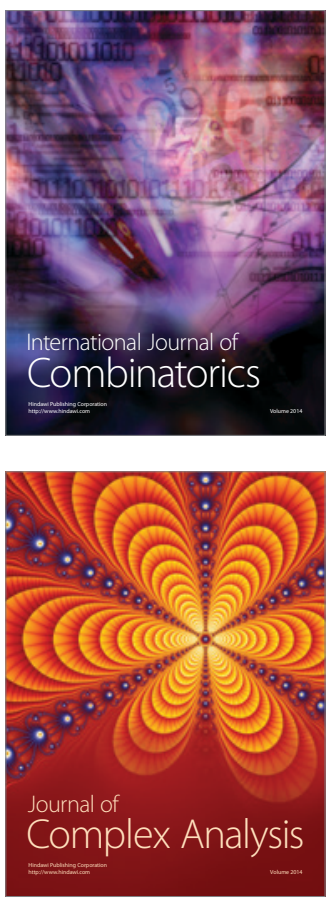

International Journal of

Mathematics and

Mathematical

Sciences
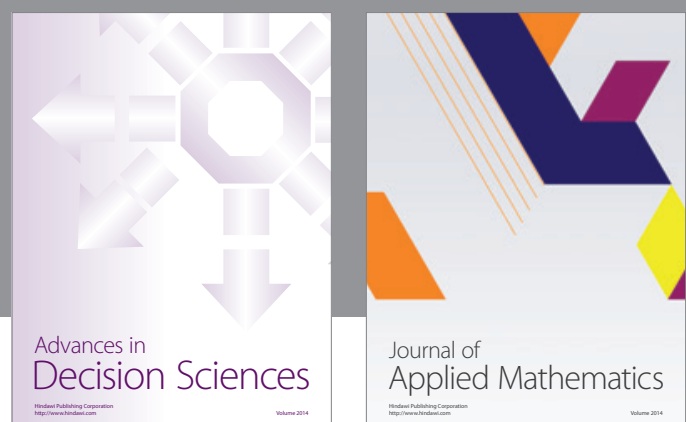

Journal of

Applied Mathematics
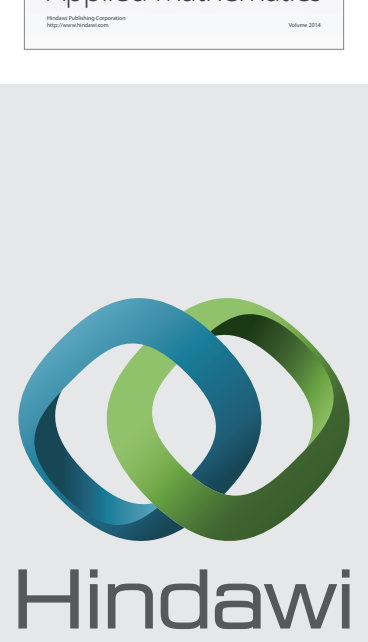

Submit your manuscripts at http://www.hindawi.com
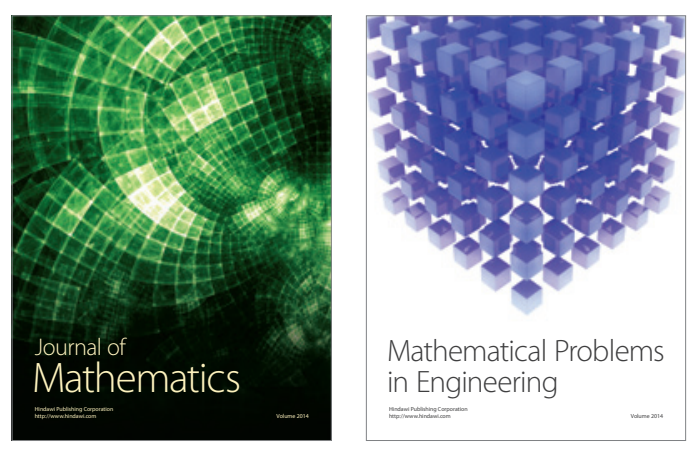

Mathematical Problems in Engineering
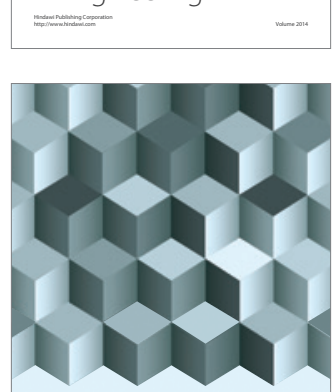

Journal of

Function Spaces
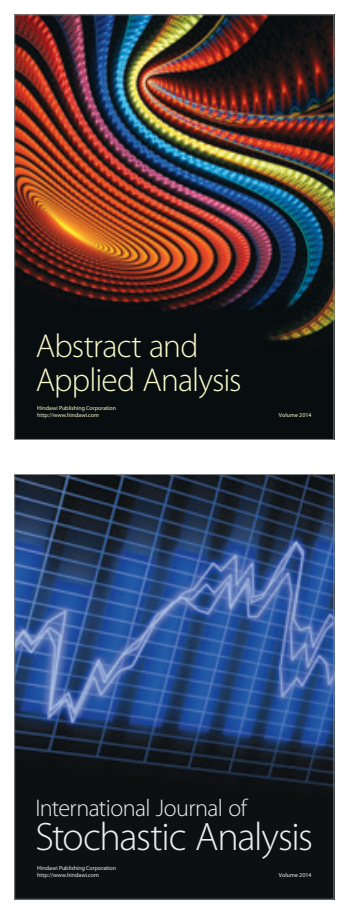

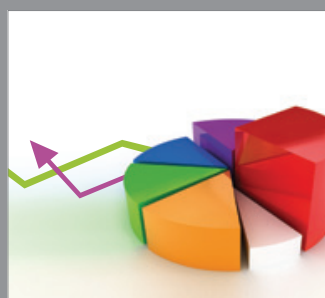

ournal of

Probability and Statistics

Promensencen
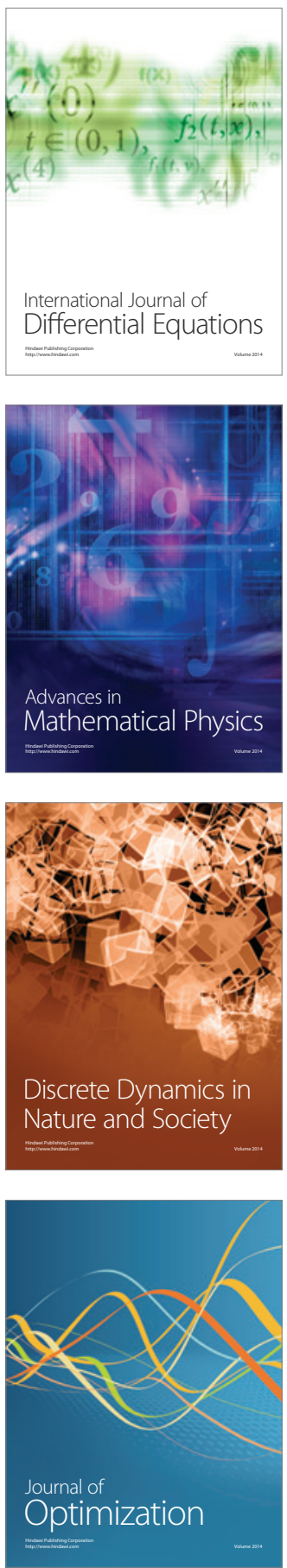